\title{
Knowledge, Attitude and Practice of Newborn Care Among Postnatal Mothers at Debre Tabor General Hospital South Gonder, Amhara Region, Ethiopia, 2018
}

\author{
Hiwot Yisak* and Amien Ewunetei \\ Department of Public Health, Debre Tabor University, Ethiopia
}

*Corresponding author: Hiwot Yisak, Department of Public Health, Debre Tabor University, Ethiopia.

\begin{abstract}
Background: Globally per year four million children die within the initial four weeks of life. And four million babies are born still.. Most neonatal deaths (99\%) occur in low financial gain and middle-income countries and concerning half the deaths occur at their home. it's tragic that several newborn die per annum which could be simply preventable by newborn care. It is calculable that concerning seventy fifth of neonatal deaths might be avoided with easy, low value tools that exist already like antibiotics for respiratory disease and infection, sterile blades to chop the point cords exploitation knit caps and marsupial care to stay babies' heat.
\end{abstract}

Objective: The objective of this study was to assess the knowledge, attitudes and practices of newborn care among postnatal mothers at Debre tabor general hospital, south Gonder Zone, Amhara region, Ethiopia 2018.

Methods: An institutional based cross-sectional study design was used for this study. Sample size was e calculated by using single population proportion formula. Final study subjects was selected using simple random sampling methods from those found in the Debre tabor general Hospital during data collection time until the sample size is fulfilled. The total sample size was 340 . The data was collected by face to face interview after the instrument is pretested and necessary modification was made. The data were entered and analyzed by Excel.

Result: A total of 186 postnatal mothers were interviewed with a response rate of $100 \%$. among them $81.2 \%$ had good knowledge $87.6 \%$ had positive attitude and $89.8 \%$ had good practice of newborn care.

Conclusion: This study indicates that nearly half and above respondents had good knowledge positive attitude and good practice of newborn care.

Abbreviations and Acronyms: AAP: American Academy of Pediatrics; ANC: Antenatal Care; BCG: Bacilli Chalmette Guerin; DPT: Diphtheria Peruses Tetanus; EDHS: Ethiopia Demographic and Health Survey; MDG: Millennium Development Goals; MD: Mean Difference; OPV: Oral Polio Vaccine; RDW: Recently-Delivered Women; UNICEF: United Nations International Children's Emergency Fun

\section{Introduction}

\section{Background information}

Neonatal wellbeing and survival depend on the care given to the neonatal, although neonatal care is an exceptionally fundamental component in decreasing child mortality, it regularly gets less than ideal attention. There have been understandings to confirm the world's commitment to progressing neonatal health. Current worldwide assessments affirm that commitment to making strides neonatal wellbeing makes important socio-economic commitments [1]. All-inclusive each year around 4 million kick the bucket within 
the to begin with 4 weeks of life. A comparative number of babies are still born. Most neonatal deaths (99\%) happen in moo wage and middle-income nations and approximately half of the deaths happen at domestic. It is awful that millions of neonatal kicks the bucket each year extraordinarily when their deaths are so effortlessly preventable. It is assessed that approximately $75 \%$ of neonatal deaths can be dodged with straightforward, moo fetched devices that as of now exist such as anti-microbials for pneumonia and sepsis, sterile edges to cut the umbilical ropes utilizing weave caps and kangaroo care to keep babies warm $[2,3]$. Each year in Africa, 30 million ladies gotten to be pregnant, and 18 million donate birth at domestic without gifted care. Each day, 3,100 newborns kick the bucket, and another 2,400 are stillborn. Too 9,600 children pass on after their to begin with month of life and sometime recently their fifth birth day each day [4].

The millennium development goal t Objectives (MDGs) had galvanized much consideration, but activity is not happening quick sufficient in Africa. Political commitment to child survival programs has spared the lives of more seasoned children, but there remains no quantifiable advance in abating neonatal death rates at the territorial level. Forty-one of each thousand babies born in Africa kick the bucket some time recently they are one month ancient. This can be the same as in Britain - more than a hundred a long time back [5]. Reductions in maternal, neonatal, and child mortality quickened amid the MDG period, particularly since 2000, most outstandingly for beneath five mortality. Tanzania met MDG 4 through a considerable lessening in mortality for children matured 1-59 months between 2000 and 2012 (normal yearly rate of lessening [ARR] 8.5\%). In any case, neonatal mortality diminished at half this rate (ARR 4.3\%)[6].

In spite of these picks up, advance remains deficiently to reach MDG 4 all-inclusive and in numerous regions, particularly in Caucasus and Central Asia, Oceania, Southern Asia and sub-Saharan Africa [3]. The worldwide under-five mortality rate has declined by more than half, dropping from 90 to 43 deaths per1,000 live births between 1990 and 2015. [7] The neonatal mortality rate fell from 36 deaths per 1,000 live births in 1990 to 19 in 2015, and the number of neonatal death's declined from 5.1 million to 2.7 million. Be that as it may, the decrease in neonatal mortality from 1990 to 2015 has been slower than that of post-neonatal under-five mortality: 47 percent compared with 58 percent universally $[2,3]$. In Ethiopia, under-five mortality has declined by 47 percent over the same period from 166 deaths per 1,000 live births to 88 deaths per 1,000 live births. Neonates who get postnatal care with within the annual rate of lessening [ARR] 8.5\%). In any case, neonatal mortality diminished at half this rate (ARR 4.3\%) [6]. Studies have shown that many newborn lives can be saved by the use of interventions that require simple technology.

The larger part of these intercessions can be viably given by a single talented birth specialist caring for the mother and the neonate. Care of all newborns incorporates quick and exhaustive drying, skin to skin contact of the neonate with the mother, line clamping and cutting after the primary minutes after birth, early start of breastfeeding, and exclusive breastfeeding [9]. By and large, genuine advance in diminishing deaths of neonatal babies in a nation with most noteworthy mortality like our own requests the next scope of ideally standard neonatal administrations with extraordinary center to the poorest portion of the populace and at the time of most prominent chance, which is at birth and within the to begin with few days of life. To diminish neonatal mortality and dismalness the practices of caring for neonatal babies must be progressed. This may be accomplished by update the information and aptitudes of those caring for babies at the time of birth and within the early postpartum period [10].

\section{Statement of the problem}

The wellbeing of the neonatal has been dismissed in spite of the tremendous number of deaths due to various reasons such as: mother's failure to look for gifted care amid conveyance, unhygienic delivery practices which result in contaminations of the neonatal, washing neonatal instantly after delivery, improper rope care, postponing quick contact between mother and neonatal since of the conviction that the neonatal is grimy and must be cleaned some time recently contact and the reality that most neonatal deaths are inconspicuous and undocumented [11].

The neonatal death's accounts for $63 \%$ of all neonatal death's and $42 \%$ of all under-five death's which makes the decrease of neonatal mortality a basic intercession. Almost 120,000 babies pass on every year within the to begin with four weeks of life. The hazard of death is most noteworthy within the to begin with 24 hours of life when more than half of death's happen and approximately threequarters of all neonatal death's happen with in the first week of life. Mediations that have the most noteworthy impact on neonatal death are less dependent on innovation and commodities than on individuals with abilities [12]. Neonatal mortality is getting to be progressively critical not as it were since the share of under-five deaths happening amid the neonatal period has been expanding, but moreover since the health interventions required to address the major causes of neonatal death's by and large contrast from those needed to address other under-five death's, and are closely connected to those that are essential to protect maternal wellbeing [3].

In spite of the fact that there have been emotional advancements in child survival, the burden of mortality within the to begin with month of life has remained for all intents and purposes unaltered. Newborns are a powerless bunch and therefore require more consideration and care. It is well set up that the welfare of a child and his future are completely subordinate upon the care and consideration gave upon him some time recently and after birth [13]. In spite of the fact that infant care is an vital mediation to diminish neonatal mortality, a ponder done at Basona Worena close 
Debre Birhan for investigating infant care behaviors appears that, the foremost as often as possible cited grouping of infant care hones detailed by moms with domestic conveyances was to tie the rope, promptly shower at that point dry the infant, hone 'Lanka mansat' (local traditional practice on newborns), provide pre-lacteal nourishing and after that start breastfeeding [14].

Another study done in Ethiopia, infant care hones detailed by recently-delivered women (RDWs) in four districts appears hones opposite to WHO suggestions that were detailed in this populace of recent mothers incorporate washing amid the primary 24 hours of life $(74.7 \%)$, application of butter and other substances to the line (19.9\%), and disposing of colostrum milk (44.5\%) [15]. To solidify picks up made in diminishing child deaths, the Government of Ethiopia bolstered by UNICEF and ELMA Philanthropies, is supporting the start of community based maternal and infant care counting administration of infant contaminations by the wellbeing expansion workers. Given moo office conveyance rates, communitybased advancement of preventive infant care practices, which has been viable in other settings, is a vital methodology. Infant care is of immense significance for the correct advancement and sound life of a child [16]. Care of the children had continuously customarily been the specialty of moms independent of education, income and social lesson contrasts. The imperative errand of parenthood is to fulfill physical, emotional, social, mental and moral needs of children. There's no question that a mother plays an imperative part in this respect.It is fundamental to assess the knowledge of moms who are reaching to beware of infant babies. Because for a newborn most visit care taker is their claim moms and it is knowledge and hones that the shapes long-standing time of the unused born [13].

The circumstance isn't distinctive within the consider range. Therefore, to guarantee the survival of newborns there's a have to be get it how the newborns are taken care of in families. In this way the show study is to survey the information and demeanor of moms with respect to infant care, discover out the hones of moms with respect to infant care in Debre tabor hospital.

\section{Objectives}

General objective: To assess knowledge, attitude and practice of newborn care among postnatal mothers in Debre tabor general hospital, Amhara region, northwest Ethiopia, from April 1-30, 2018.

Specific objectives: To determine the knowledge of newborn care among postnatal mothers in Debre tabor general hospital, Amhara region, northwest Ethiopia, 2018.

To determine attitude regarding newborn care among postnatal mothers in Debre tabor general hospital, Amhara region, northwest Ethiopia, 2018.

To describe the practice of newborn care among postnatal mothers in Debre tabor general hospital, Amhara region, northwest Ethiopia, 2018.

\section{Method}

\section{Study area and period}

This study was be conducted in march 2018 at Debre Tabor General Hospital $\{\mathrm{DTGH}\}$, the only general hospital in South Gonder Zone, Amhara Regional, state of North west Ethiopia and it is specifically found in Debre Tabor town, which is the capital city of South Gonder zone and it is far $97 \mathrm{~km}$ from Bahir Dar the capital city of Amhara regional state and $666 \mathrm{~km}$ far from Addis Ababa which is the capital city of Ethiopia.

Debre Tabor is bordered on the East by Abaregay Kebelle, on the South by Debre Tabor Eyesus Church, on the west by Tsegur Adiko Kebelle and on the North by Weybla Kebelle. The town has four kebeles and its total population according to 2007 population census is 83,081 of whom, 43,301(52.12\%) Female and $39,780(47.88 \%)$ male. In this town there are eight Orthodox Churches, one Mosque and 3 Protestant Churches.

The climatic condition of the town is Weyna Dega and its average annual rain fall, and temperature is $1553.7 \mathrm{~mm}$. In this town there are three public Health center and one general hospital and more than two private clinics. Debre Tabor General Hospital was established by Dr Augambi in 1923 E.C and totally under control by the government in 1969 E.C. The total area is 94,725 meter square. There are more than 30 services delivered in this hospital like mother and child health (MCH) unit, gynecology and obstetrics unit, psychiatric clinic, laboratory unit, pharmacy unit, emergency care unit, ophthalmic service unit, minor operation unit, labor ward, internal medicine ward, neonatal intensive care unit (NICU), out - patient department (OPD), dentist unit, surgical ward and pediatrics ward. The study was conducted on April (1-30).

\section{Study design}

Institution based cross sectional study design was be conducted.

\section{Population}

Source population: All postnatal mothers attending in Debre tabor general hospital, Ethiopia.

Study population: All postnatal mothers attending in Debre tabor general hospital, Ethiopia during data collection period.

Inclusion Criteria: Post-natal mothers of babies born normal and alive.

Exclusion criteria: Postnatal mothers who are unconscious, seriously ill and mentally retarded.

\section{Sample size and sampling technique}

Sample size determination: The sample size was determined by using a formula for estimating a single population proportion with confidence interval of 95\%, 5\% marginal error, and 5\% no response rate, a total of 186 mothers were required for the study. The prevalence of good practice of new born care among post- 
natal mothers in Addis Ababa university 60.1\% [28]. The data was collected from each mother in the sample.

Sample Size Determination:

$\mathrm{n}=\mathrm{z} 2 * \mathrm{p}(1-\mathrm{p}) / \mathrm{d} 2$

Where

$\mathrm{n}=$ sample size needed

$\mathrm{d}=$ desired precision $(5 \%)$

$\mathrm{p}=$ proportion of population $60.1 \%$

$\mathrm{Z} / 2=1.96$ at confidence level $95 \%$.

Given

$\mathrm{P}=0.6$

$\mathrm{Q}=1-0.6=0.4$

$\mathrm{D}=0.05$

$Z=1.96$

$\mathrm{n}=\mathrm{z} 2 . \mathrm{pq} \backslash \mathrm{d} 2=(1.96) 2 \times 0.6(1-0.6) \backslash(0.05) 2=368.79$

since my source of population is less than 10000 which is 340 .....then used reduction formula as follows

$\mathrm{nf}=\mathrm{n} /(1+\mathrm{n} / \mathrm{N})=369 /(1+369 / 340)=177$

Which is $\mathrm{n}=$ sample size

nf=final sample size

$\mathrm{N}=$ total no of post-natal mother .Then by considering $5 \%$ nonresponse rate

nf become $177+9=186$

Sampling Technique: The total sample size was draw postnatal mother randomly who are going to be included in the study and direct interview technique using prepared questionnaire during post-natal service used. Considering average client who was come to hospital per day for PNC service is seventeen women and considering 20 working day in one month the total number of post-natal mothers attending post-natal care service within a month is $17 \times 20=340$ therefore total number of women attending PNC in DTGH was 340 and 186 study participants was selected using simple random sampling method. The service was given in all working days.

\section{Operational definition}

Care of newborn: It is the care given to the neonatal following birth like: providing warmth

\& prevention of heat loss, initiation of breast feeding, protection from infections, safe umbilical cord tie and cutting and postponing bathing for 24 hours.

Knowledge: Factual information that the respondents knows regarding the newborn care.
- Good knowledge: Those answer greater than $60 \%$ question out of total knowledge related question.

- $\quad$ Poor knowledge: Those answer less than $60 \%$ question out of total knowledge related question.

Attitude: The feeling and beliefs of respondents with regard to newborn care.

- Positive Attitude: who answer positive greater than $60 \%$ of attitude related question.

- $\quad$ Negative Attitude: who answer positively less than $60 \%$ of attitude related question.

Practice: The intended actions that the respondents done or do to give care for the newborn baby.

- Good practice: score greater than $60 \%$ on the overall practice question

- $\quad$ Poor practice: Those who answer less than $60 \%$ of practice related question.

\section{Data collection procedure}

Structured questionnaire was developed based on the independent variable and factors identified though literature review. Before the data collection, questionnaires was be pre-tested out of the selected mother to make sure that the questions are clear and could be understood by the study participants and $5 \%$ of postnatal women was be requested to respond to this questionnaire. In order to avoid response biases, the participant in the pilot study was not included in the main survey. After review of the questionnaire based on the pretest findings, the final questionnaire was used to interview the selected mothers.

Four data collector was trained regarding the objective, methods of data collection and other related and assigned at PNC ward .For better management of data collection process one data collector who is working at the study area was be recruited in consultation with their immediate supervisors based on their experience and good client relationship.

\section{Data quality control}

Copy of the questionnaire was be given to advisers to be examined whether the number and type of the items in the questionnaire can measure the concept or objective of the study and by taking suggestion necessary amendment was be made.

The data collection tool was be translated to the local language (Amharic) to make the questions easily understandable and it was be retranslated back to the English language to evaluate its consistency. All the data collectors was be the experienced and trained who are not working in the delivery and postnatal ward during data collection period. Training was be given for the data collectors focusing on the questionnaire content to ensure consistency of data, obtaining consent, maintaining neutrality, privacy issues, personal relation and ethics in research for one-day duration. 
Filled questionnaires was be checked daily for completeness and consistency of the responses to eliminate possible errors.

\section{Data processing, analysis and presentation}

The collected data was be checked manually for completion and any incomplete or misfiled Questions. Tally of the data was done, and frequency and percentage was done. Results was compiled and presented using tables, graphs and texts.

\section{Ethical consideration}

Ethical clearance and approval was obtained from department of midwifery, college of health science, Debre Tabor University. After explaining the objectives of the study in detail, informed verbal consent was sought from all study participants (the postnatal mothers). All the participants was reassured of the anonymity, and as personal identifiers was not be used. Then, after obtaining informed consent from every participant, the data collectors was continuing the job by giving due respect to the norms, values, beliefs, culture, and ensured the confidentiality of the data.

\section{Dissemination of the result}

The study result was disseminated through Debre Tabor University, Debre tabor general hospital and Amhara Regional Health bureau.

\section{Result}

The study results including sociodemographic, antenatal and birth history, knowledge, attitude and practice of newborn care. A total of 186 postnatal mothers were interviewed and the response rate was $100 \%$ (Table $1 \& 2$ ).

Table 1: Distribution of sociodemographic characteristics of postnatal mothers in Debre tabor general hospital, South Gonder zone, 2018.

\begin{tabular}{|c|c|c|}
\hline Variable & Frequency $(n=186)$ & Percent \% \\
\hline \multicolumn{3}{|c|}{ Age of the Mother in Year } \\
\hline \multicolumn{3}{|l|}{$15-19$} \\
\hline $20-24$ & 68 & 36.56 \\
\hline $25-29$ & 95 & 51.1 \\
\hline $30-34$ & 13 & 6.989 \\
\hline $35-39$ & 4 & 2.2 \\
\hline Marital status single & 12 & 6.5 \\
\hline Married & 153 & 82.3 \\
\hline Divorced & 21 & 1 \\
\hline \multicolumn{3}{|c|}{ Mother 's Religion } \\
\hline Orthodox & 160 & 86 \\
\hline Protestant & 8 & 4.3 \\
\hline Muslim & 18 & 9.7 \\
\hline \multicolumn{3}{|l|}{ Ethnicity } \\
\hline Amhara & 186 & 100 \\
\hline
\end{tabular}

Table 2: Distribution of sociodemographic characteristics of mothers in Debre tabor General Hospital south Gonder zone, Ethiopia, 2018.

\begin{tabular}{|c|c|c|}
\hline Variable & Frequency (n=186) & Percent \% \\
\hline & Mother's Level of Education & 10.8 \\
\hline No unable to read and write & 20 & 11.3 \\
\hline Read \& write & 21 & 12.9 \\
\hline Primary (1-4) & 24 & 16.9 \\
\hline Primary (5-8) & 30 & 24.7 \\
\hline Secondary (9-12) & 46 & 24.2 \\
\hline Tertiary (>12) & 45 & 31.7 \\
\hline Housewife & Mother's Occupation & 45.2 \\
\hline Government employee & 59 & 18.8 \\
\hline Merchant & 34 & 4.3 \\
\hline Student & 8 & \\
\hline
\end{tabular}




\begin{tabular}{|c|c|c|}
\hline $651-1400$ & 35 & $18.80 \%$ \\
\hline $1401-2350$ & 78 & $41.90 \%$ \\
\hline $2351-3550$ & 18 & $9.60 \%$ \\
\hline $3551-5000$ & 22 & $11.80 \%$ \\
\hline$>5000$ & 28 & $15.10 \%$ \\
\hline
\end{tabular}

The minimum and maximum age of 18 and 39 respectively. Most of the study participants $95(51.1 \%)$ were in the age group of 25-29 years, and the least $4(2.15 \%)$ were in the age group 35-39 years. Majority of respondents 153(82.7\%) were married, 160(86\%) were orthodox by religion, $186(100 \%)$ were Amhara by ethnicity, $46(24.1 \%)$ had secondary (9-12) education, 84(45.2\%) were government employee and out of the total respondents $78(41.9 \%)$ of them had a monthly family income ranging 1401-2350 Et, birr (based on the Ethiopian employment income/per month payroll rate).
Out of the total postnatal mothers interviewed, 184(98.9\%) had attended antenatal clinic and from those postnatal mothers who had attended ANC clinic, $150(80.6 \%)$ of them started their first visit at 3-6 month of gestation. 136(73.1\%) of mothers had four ANC visit during pregnancy. Majority of the mothers were multiparous $125(67.2 \%)$ while primiparous accounted for $61(32.8 \%)$ of those interviewed. most respondents $135(72.6 \%)$ planed where to deliver their baby and 176(94.6\%) of newborns had a birth weight ranging 2.5-4 kilograms. Male neonatal accounts 82(44.1\%) while female were 104(55.9\%) (Figure 1).

Figure 1: Distribution of respondents by information on newborn care received, in Debar Tabor General Hospital South Gonder Zone Ethiopia 2018.

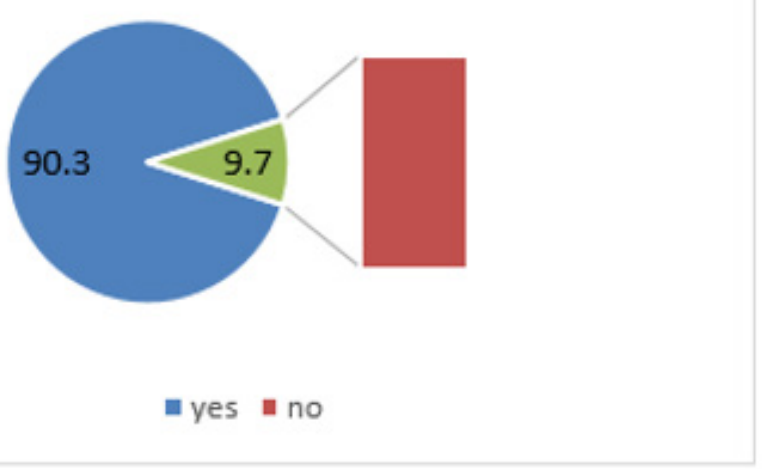

Of the total interviewed postnatal mothers, 168(90.3\%) of them had received different information regarding newborn care and the remaining $18(9.7 \%)$ of them not received information (Table 3).

Of those who had received information/education regarding newborn care, $90.3 \%$ of them mentioned provider of information on newborn care, 168 (90.3\%) were nurses and responds mentioned provider of information were family 78 (41.9\%). Regarding the type of information, $82.7 \%$ of respondents mentioned breastfeeding 178 (96.2\%)Thermoregulation and followed by $89.8 \%$ of respondents mentioned immunization (Table 4\&5).

Table 3: Type and source of information on newborn care received, among postnatal mothers in Debre tabor General hospital, 2018.

\begin{tabular}{|c|c|c|c|}
\hline Variable & Response & Frequency & Percent of Cases \\
\hline \multirow{5}{*}{ Type of information received* } & Breastfeeding & 154 & $82.70 \%$ \\
\hline & Cord care & 115 & $61.80 \%$ \\
\hline & Eye care & 78 & $41.90 \%$ \\
\hline & Thermoregulation & 178 & $96.20 \%$ \\
\hline & Immunization & 167 & $89.80 \%$ \\
\hline \multirow{5}{*}{ Provider of information on newborn care* } & Doctor & 8 & $4.30 \%$ \\
\hline & Nurses & 168 & $90.30 \%$ \\
\hline & Family & 78 & $41.90 \%$ \\
\hline & $\begin{array}{l}\text { Media (e.g. pamphlets, brochures, maga- } \\
\text { zines) }\end{array}$ & 35 & $18.80 \%$ \\
\hline & Peers/ friends & 5 & $2.70 \%$ \\
\hline
\end{tabular}


Table 4: Knowledge on newborn care of postnatal mothers in Debre tabor General Hospital 2018.

\begin{tabular}{|c|c|c|}
\hline Variable & Frequency $(\mathrm{N}=186)$ & Percent \% \\
\hline \multicolumn{3}{|c|}{ Thermoregulation } \\
\hline \multicolumn{3}{|l|}{ Baby should be kept warm after birth } \\
\hline Yes & 151 & $81.20 \%$ \\
\hline No & 35 & $18.80 \%$ \\
\hline \multicolumn{3}{|l|}{ Method used to keep warm a Newborn } \\
\hline Skin to skin contact & 62 & $33.30 \%$ \\
\hline Wrap the baby using clean cloth immediately & 43 & $23.10 \%$ \\
\hline Both $1 \& 2$ & 46 & 24.7 \\
\hline First bath after delivery Immediately & 53 & $28.50 \%$ \\
\hline After six hours & 2 & $1.10 \%$ \\
\hline After one day & 123 & $66.10 \%$ \\
\hline Don't know & 8 & $4.30 \%$ \\
\hline \multicolumn{3}{|c|}{ Cleanliness and Cord Care } \\
\hline Instrument used to cut the cord after delivery New blade & 180 & $96.80 \%$ \\
\hline Don't know & 6 & $3.20 \%$ \\
\hline After the cord is cut, umbilical stump Cover with cloth & 28 & $15.10 \%$ \\
\hline Uncover, keep dry and clean & 121 & $65.10 \%$ \\
\hline Don't know & 37 & $19.90 \%$ \\
\hline Soiled umbilical stump can be cleaned with Clean with water & 164 & $88.20 \%$ \\
\hline Apply alcohol & 8 & $4.30 \%$ \\
\hline Other ( using dry cloth, tissue paper) & 14 & $7.50 \%$ \\
\hline Any substances can be applied on the umbilical stump Yes & 12 & $6.50 \%$ \\
\hline No & 174 & $93.50 \%$ \\
\hline
\end{tabular}

Table 5: knowledge on newborn care among postnatal mothers in Debre tabor General hospital, 2018G.C Breastfeeding.

\begin{tabular}{|c|c|c|}
\hline Breastfeeding & Frequency $(\mathrm{N}=186)$ & Percent \% \\
\hline \multicolumn{3}{|c|}{ Time of breastfeeding initiation } \\
\hline$<30$ minutes & 102 & $54.80 \%$ \\
\hline 30 minutes- 1 Hour & 35 & $18.80 \%$ \\
\hline After 1 hour & 40 & $21.50 \%$ \\
\hline Don't know & 9 & $4.80 \%$ \\
\hline
\end{tabular}

Most of the respondents 151(81.2\%) were aware of the need of newborn baby to be kept warm at birth and of these mothers' the modes of keeping the baby warm identified are shown in table 3. Majority of the respondents (66.1\%) replied time of newborns first bathing is after one day of birth and $53(28.5 \%)$ of them said that newborn baby bathed immediately.

Among the study subjects, 121 (65\%) of them correctly stated that the stump should be uncovered, kept clean \& dry and most 180 (96.8\%) of mothers knew that new blade is used to cut the cord after delivery. Out of those interviewed, 164 (88.2\%) of mothers believed that clean water should be used to clean the soiled umbilical stump while 8 (4.3\%) believed alcohol should be used. Majority of mothers 174 (93.5\%) replied that any substance can't be applied on the umbilical stump.

The overall knowledge score of the study participants shows that majority $151(81.2 \% \%)$ of the respondents had good knowledge and the remaining $35(18.8 \% \%)$ had poor knowledge (Table 6).

Table 6: Postnatal mother's attitude towards newborn care, in Debera tabor General Hospital 2018.

\begin{tabular}{|c|c|c|c|c|c|c|}
\hline \multirow{2}{*}{ Variable } & \multicolumn{2}{|c|}{ Disagree } & \multicolumn{2}{|c|}{ Neutral } & \multicolumn{2}{|c|}{ Agree } \\
\hline & $\mathbf{N}$ & $\%$ & $\mathbf{N}$ & $\%$ & $\mathbf{N}$ & $\%$ \\
\hline Newborn babies lose their body heat fast & 98 & 52.3 & 12 & 6.5 & 76 & 40.9 \\
\hline Mother baby skin to skin contact prevents the baby from getting cold & 18 & 9.7 & 5 & 2.7 & 163 & 87.6 \\
\hline
\end{tabular}




\begin{tabular}{|c|c|c|c|c|c|c|}
\hline Newborn baby can be bathed immediately after birth & 113 & 60.8 & 23 & 12.4 & 50 & 26.9 \\
\hline $\begin{array}{l}\text { Previously used razor blade can be washed and used to cut the umbilical } \\
\text { cord of the baby }\end{array}$ & 176 & 94.6 & 6 & 3.2 & 4 & 2.2 \\
\hline Dirty umbilical cord can cause infection of the baby & 5 & 2.7 & 3 & 1.6 & 178 & 95.7 \\
\hline
\end{tabular}

Most of the study participants were agreed on as newborns lose their body heat faster, skin to skin contact prevents the baby from getting cold, dirty umbilical cord can cause infection to the baby $40.9 \%, 87.6 \%, \& 95.7 \%$ respectively.

Also, majority of the respondents were disagreed on newborn baby can be bathed immediately after birth 113 (60.8\%), a previously used razor blade can be washed and used to cut the umbilical cord of the baby 176 (94.6\%).

The overall attitude score of the study participants shows that majority $163(87.6 \% \%)$ of the respondents had positive attitude and the remaining 23 (12.4\%) had negative attitude (Table 7).

Table 7: Distribution of respondents by practice of newborn care among postnatal Mothers in Debre tabor General Hospital 2018.

\begin{tabular}{|c|c|c|}
\hline Variables & Frequency $(\mathrm{N}=186)$ & Percent (\%) \\
\hline Baby placed when delivered On the mother's abdomen & 156 & 83.9 \\
\hline On clean surface & 30 & 16.1 \\
\hline Instrument used to cut the umbilical cord New or boiled blade & 186 & 100 \\
\hline \multicolumn{3}{|c|}{ Substance Applied on the Stump } \\
\hline No & 186 & 100 \\
\hline \multicolumn{3}{|c|}{ Method Used to Keep Baby Warm } \\
\hline Skin to skin contact & 75 & 40.3 \\
\hline Wrapped the baby in a cloth immediately & 80 & 43 \\
\hline Both & 31 & 16.7 \\
\hline
\end{tabular}

Mothers responded that baby was placed on the mother's abdomen and a clean surface accounts $156(83.9 \%)$ and $30(16.1 \%)$ respectively before the placenta was expelled. The umbilical cord was cut with a new or boiled blade in 186 (100\%) deliveries. all $(100 \%)$ replied as nothing was applied on the umbilical stump.

Concerning maintenance of warm chain for the newborn 75 $(40.3 \%)$ of the respondents were used skin to skin and $80(43 \%)$ wrapped using dry clean cloth to keep their baby warm.

The overall practice score of the study participants shows that majority 167 (89.8\%) of the respondents had good practice and the remaining $19(10.2 \%)$ had poor practice.

\section{Discussion}

In this think about out of the whole respondents met, 151 (81.2\%) of the respondents have good knowledge of infant care and $163(87.6 \%)$ of the respondents had positive state of mind to wards unused born care This ponder is marginally higher than a think about done at Addis Ababa 55.3\% Madurai, Tamil Nadu India by which $61 \%$ of respondents had favorable demeanor [21]. This disparity may be due to the truth that there's sociocultural distinction, little test measure, think about plan, and think about period. Moreover, the overall practice score of the consider members appears that larger part 167 (89.8\%) of the respondents had good hone, which is higher than the finding of a consider done at, Addis Ababa $60.6 \%$ Mandurah Locale, Northwest Ethiopia, where (40.6\%) of them had great infant care hone [34].
This distinction can be due to an expanded wellbeing administrations and extraordinary intercession centering on child health. Regarding information of warm upkeep, 151 (81.2\%) of respondents expressed the require of keeping the infant warm. Approximately washing time, this consider uncovered that 53 $(28.5 \%)$ of them replied time of to begin with showering an infant is after one day of birth, 121(65.1\%) of them correctly stated that the stump ought to be revealed, kept clean \& dry and 180 (96.6\%) of moms knew that unused edge is utilized to cut the line after conveyance. This figure is nearly comparable with a think about done in northern Cameroon, which appears the utilize of sterile fabric for cutting umbilical line was reported by 307 (88.5\%) mother [23]. The umbilical line was cut with an unused bubbled edge in 262 (63.3\%) deliveries.

Concerning upkeep of warm chain for the infant nearly all of the respondents were used either skin to skin or wrapped utilizing dry clean cloth to keep their infant warm, which distinctive from a ponder done at Southern Tanzania Skin-to-skin contact between mother and infant after delivery was once in a while practiced [29]. This can be may be due to think about put and period contrast. Larger part of the respondents 123 (66.1\%) of the respondents said they washed their infant after one day of birth and 53 (28.5\%) of them said that infant child showered quickly. This finding is lower than the finding in a study done at Garoua city Northern Cameroon, which is six hours deferred to begin with shower was given by 244 (70.3\%) mothers. The contrast may be due to consider period contrast and an increase of mediations by diverse organizations 
working on child wellbeing .With respect to breastfeeding 137 (73.6\%) newborns were started breastfeeding inside an hour after delivery. This finding is lower than a think about done at Southern Tanzania, which is $83 \%$ of women breastfed inside 24 hours of convey. This inconsistency may be due to the difference of consider period, test estimate and range [23].

\section{Strength \& Limitations of The Study}

\section{Strengths of study}

Recall bias was minimized since the respondents were postnatal mothers.

\section{Study limitations}

Qualitative method to support the quantitative findings was not used. Therefore, there was a risk that mothers may report what was expected of them, but their actual practices may be different.

Study design was cross sectional so that cause and effect relationship of variables was difficult to know which one is came first and which came last.

\section{Conclusion}

This study indicated that nearly half and above respondents had good knowledge, positive attitude and good practice of newborn care.

The awareness and knowledge among mothers regarding newborn care need to be enhanced in order to achieve better targets in neonatal and under-five mortality and morbidity.

\section{Acknowledgement}

None.

\section{Conflict of Interest}

No conflict of interest.

\section{References}

1. Darmstadt GL, Kinney MV, Chopra M, Cousens S, Kak L, et al. (2014) Who has been caring for the baby? Lancet 384(9938): 174-88.

2. Bogale Worku, Mulualem Gessesse (2012) Federal Ministry of Health Ethiopia, Newborn Care training (August): 1-102.

3. You D, Hug L, Ejdemyr S, Beise J, Idele P, et al. (2015) Levels \& Trends in Child Mortality Report 2015.

4. The Partnership For Maternal \& Child Health (2010) Opportunities for Africa's Newborns 250.

5. Joy L, Kerber K Opportunities for Africa's newborns: Practical data, policy and programmatic support for newborn care in Africa. Lancet 327(8493): 1331-1332.

6. Afnan-Holmes H, Magoma M, John T, Levira F, Msemo G, et al. (2015) Tanzania's Countdown to 2015: an analysis of two decades of progress and gaps for reproductive, maternal, newborn, and child health, to inform priorities for post-2015. Lancet Glob Health 3(7): e396-409.

7. United Nations (2015) New York 2015. The Millennium Development Goals Report 2015.
8. JSI L10K (2014) News from the Last Ten Kilometers 2(4): 2-5.

9. WHO (2015) Newborn care at birth. World Health Organization.

10. Shrestha T, Bhattarai SG, Silwal K (2013) Knowledge and Practice of Postnatal Mother in Newborn Care. JNMA J Nepal Med Assoc 52(190): 372-377.

11. Nations U (2014) The Millennium Development Goals Report 2014 United Nations pp. 59.

12. Survey H (2011) Ethiopia Demographic and Health Survey, 2011. Heal San Francisco, USA, pp. 51.

13. Castalino F, Nayak BS, Souza DA (2014) Knowledge and Practices of Postnatal Mothers on Newborn Care in Tertiary Care Hospital of Udupi District. Nitte University Journal of Health Science 4(2): 98-101.

14. Salasibew MM, Filteau S, Marchant T (2014) A qualitative study exploring newborn care behaviors after home births in rural Ethiopia: implications for adoption of essential interventions for saving newborn lives. BMC Pregnancy Childbirth 14: 412.

15. Callaghan-Koru JA, Seifu A, Tholandi M, de Graft-Johnson J, Daniel E, et al. (2013) Newborn care practices at home and in health facilities in 4 regions of Ethiopia. BMC Pediatr 13(1): 198.

16. Unicef (2004) What Works for Children in South Asia NEWBORN CARE : AN OVERVIEW.

17. Asif M, Vishnu BB, Ekambaram M (2010) Knowledge attitiude and practice of neonatal care among postnatal mothers in a tertiary care hospital, in South India. Curr Pediatr Res 14(2) :147-152.

18. Angel Rajkumari G (2015) Assessing the Knowledge and Practice Regarding Newborn Care among Postnatal Mother in Nirmala Hospital Suryapet, Telangana, India. Glob J Curr Res 3(4): 106-110.

19. Rama R, Gopalakrishnan S, Pm U (2014) Assessment of knowledge regarding new-born care among mothers in Kancheepuram district Tamil Nadu. Int J Community Med Public Heal 1(1): 58-63.

20. Sharafi R, Esmaeeli H (2013) Knowledge assessment of neonatal care among postnatal mothers. Iran J Neonatol 4(1): 28-31.

21. Francisca M, Mireille EM, David C, Pascal F, Christopher K (2013) Mothers' Knowledge and Practice on Essential Newborn Care at Health Facilities in Garoua City, Cameroon. Health Sci Dis 14(2): 1-6.

22. Gupta P, Srivastava V, Kumar V, Jain S, Masood J, et al. (2010) Newborn Care Practices in Urban Slums of Lucknow City, UP. Indian J Community Med 35(1): 82-85

23. Darling B, Ranjita S, Wankhede, Benjamin BA (2014) Knowledge, attitude and practice of postnatal mothers regarding newborn care in selected maternity centers in Madurai. Int J Allied Med Sci Clin Res 2(2): 119-124.

24. Bhatt B, Malik JS, Jindal H, Sahoo S, Sangwan K (2015) A study to assess cord care practices among mothers of newborns in urban areas of Rohtak Haryana. Online Int J 5(1): 55-60.

25. Kokebie T, Aychiluhm M, Degu G (2015) Community Based Essential New Born Care Practices and Associated Factors among Women in the Rural Community of Awabel District, East Gojjam Zone, Amhara, Ethiopia, 2013. Int J Adv Sci Res 1(01): 17-27.

26. Tegene T, Andargie G, Nega A, Yimam K (2015) Newborn Care Practice and Associated Factors among Mothers who gave Birth within One Year in Mandura District, Northwest Ethiopia. Clinics Mother Child Health 12(1): 1-7. 